\title{
Constructing Novel Fiber Reinforced Plastic (FRP) Composites through a Biomimetic Approach: Connecting Glass Fiber with Nanosized Boron Nitride by Polydopamine Coating
}

\author{
XueMei Wen, ${ }^{1,2}$ ZaoZao Xiao, ${ }^{1}$ Tao Jiang, ${ }^{1}$ Jian Li, ${ }^{1}$ \\ Wei Zhang, ${ }^{1}$ Lei Zhang, ${ }^{1,3}$ and Huaiqi Shao ${ }^{1}$ \\ ${ }^{1}$ School of Material Science and Chemical Engineering, Tianjin University of Science and Technology, Tianjin 300457, China \\ ${ }^{2}$ Tianjin Synthetic Material Research Institute, Tianjin 300220, China \\ ${ }^{3}$ College of Marine Science and Engineering, Tianjin University of Science and Technology, Tianjin 300457, China
}

Correspondence should be addressed to Jian Li; lijian@tust.edu.cn

Received 8 May 2013; Accepted 11 November 2013

Academic Editor: Honggang Zhu

Copyright (C) 2013 XueMei Wen et al. This is an open access article distributed under the Creative Commons Attribution License, which permits unrestricted use, distribution, and reproduction in any medium, provided the original work is properly cited.

\begin{abstract}
A biomimetic method was developed to construct novel fiber reinforced plastic (FRP) composites. By mimicking mussel adhesive proteins, a monolayer of polydopamine was coated on glass fiber (GF) surface. The polydopamine-treated GF (D-GF) adsorbed boron nitride $(\mathrm{BN})$ nanoparticles, while obtaining micronano multiscale hybrid fillers BN-D-GF. Scanning electron microscopy (SEM) results showed that the strong interfacial interaction brought by the polydopamine benefits the loading amount as well as dispersion of the nano-BN on GF's surface. The BN-D-GF was incorporated into epoxy resin to construct "FRP nanocomposites." The morphology, dynamic mechanical and thermal characteristics of the FRP nanocomposites were analyzed. SEM morphology revealed that BN-D-GF heterogeneous dispersed in epoxy matrix. There was good adhesion between the polymer matrix and the BN-D-GF filler. The dynamic modulus and mechanical loss were studied using dynamic mechanical analysis (DMA). Compared with neat epoxy and untreated GF reinforced composites, BN-D-GF/epoxy and D-GF/epoxy systems showed improved mechanical properties. The thermal conductivity, Shore D hardness, and insulation properties were also enhanced.
\end{abstract}

\section{Introduction}

Epoxy resins are a class of high performance thermosetting polymers for application in the automotive, construction, and aerospace industries. High specific stiffness, dimensional stability, lightweight, high corrosion resistance, and excellent insulating properties make them valuable in many electrical research areas, especially for insulated packing [1-3]. Recently, using epoxy resin as the matrix for fiber-reinforced plastic (FRP) composites has been increased significantly [46]. The FRP composites are considered as ideal candidates for academic research as well as industry applications because of their improved physical/chemical properties. Glass fiber (GF) is usually used for preparing large varieties of FRP composites [7]. The principal advantages of GF are low cost, high strength, and modulus.
For FRP composites, the extent of adhesion of polymer matrix to the reinforcing fibers is very important. It is well known that stress passes from the fibers to the matrix through the interface. Therefore, the adhesive force affects the strength and rigidity of the reinforced plastics and their fracture behavior [8]. In order to enhance affinity between GF and polymer matrix, modification of the GF surface can be done by (a) coating fiber with a silane with reactive end groups [9], (b) coating fiber with a rubber emulsion [10], (c) coating fiber with a solution of different polymers such as polyurethane and polystyrene [11], and (d) blending with nanoscopic materials, such as carbon nanotube [12], graphene [13], and inorganic oxide nanoparticles [14].

Filling epoxy resin with nanomaterials, especially thermally conductive nanofillers $\left(\mathrm{Al}_{2} \mathrm{O}_{3}\right.$ [15], $\mathrm{AlN}$ [16], $\mathrm{BN}$ [17]), 
is emerged as an effective way to enhance the rather poor thermal conductivity of epoxy resin, because the heat dissipation in microelectronic packaging is becoming increasingly important as the demands in denser and faster circuits intensify. For nanofillers, their dispersion and interfacial interactions with epoxy resin matrix are the most crucial factor affecting the optimal properties of the nanocomposites $[18,19]$. Nanofillers should be well dispersed in the matrices to obtain a high aspect ratio and large interfacial area between the phases, whereas strong interfacial interactions between the nanofillers and epoxy resin matrices would facilitate the stress transformation and thermal conductivity.

Many previous researches were interested in combining the advantages of microscale fibers and nanoscale particles. For example, Wan et al. reported that multiscale reinforcements consisting of clay and short glass fiber filled polyamide 6 were prepared by a two-step melt compounding process $[20,21]$. The synergy of multiscale reinforcements of clay and glass fiber provided better physical properties for polymer-matrix composites than each of the individual fillers. Besides the above methods based on physically blending microscale fibers and nanoscale fillers [15-17, 20, 21], a more effective method is to disperse nanofillers onto glass fibers and then construct novel "FRP nanocomposites." The advantages include (1) a better dispersion of thermally conductive nanofillers in polymer matrix, (2) an enhanced affinity between fiber and matrix, and (3) enhancing performance of FRP composites without marked increase in cost due to small amount of nanofillers. Traditional method is to modify their surface in the presence of functional coupling agents, such as silane coupling agents [22-24]. However, the amount of active groups on nanofillers or glass fibers is usually very small, and the reactions require strict chemical conditions. Furthermore, the main caveat of this strategy is that the coupling agents are often material specific and thus lack efficacy across a broad range of FRP nanocomposites.

In nature, marine mussel adhesive proteins (MAPs) contain large amounts of a particular amino acid known as 3,4-dihydroxy-L-phenylalanine (dopamine, DOPA) and have attracted great attention owing to the amazing ability of mussels to adhere to various kinds of surfaces $[25,26]$. In 2007, Lee et al. reported polydopamine (PDOPA) adhesive coatings on a wide variety of material surfaces including noble metals, oxides, polymers, semiconductors, and ceramics prepared by mimicking MAPs [25].

Inspired by these works, we hypothesized that if a catechol-rich polymer layer can be prepared as the interface between nanofillers and GF, the interfacial interactions may be significantly enhanced and hence the physical/chemical properties of the FRP composites can be improved at very low nanofillers loadings. To verify the hypothesis, we prepared dopamine-modified glass fiber (D-GF) via a facile waterassisted process and then coated $\mathrm{BN}$ nanoparticles on the surface. The novel reinforcement (BN-D-GF) was incorporated into an epoxy resin to form FRP nanocomposites. Specifically, the structure, morphology, and thermomechanical properties of these novel materials were in detail investigated.

\section{Materials and Methods}

2.1. Materials. Epoxy resin based on diglycidyl ether of bisphenol A (DGEBA, E51) and triethylenetetramine (TETA) curing agent were purchased from Jiangtian Reagent Chemicals Co. Ltd. Short-cut glass fibers were supplied by Owens Corning Chemicals and 3,4-dihydroxyphenethylamine hydrochloride (DOPA, 98\%) was purchased from SigmaAldrich, respectively. All the other chemicals were purchased locally and of analytical reagent grade.

\subsection{Preparation of Modified Glass Fibers and FRP Composites.} In a typical experiment, $5 \mathrm{~g}$ short-cut glass fiber (GF) was mechanically stirred in $500 \mathrm{~mL} 10 \mathrm{mM}$ Tris buffer solution (pH 8.5) [25]. DOPA (1 g) was then added and the suspension was stirred for $8 \mathrm{~h}$ at room ambient condition. The suspension was then centrifuged at $2500 \mathrm{rpm}$ for $3 \mathrm{~min}$ and then the dopamine coated glass fiber (D-GF) was obtained. D-GF was redispersed in $500 \mathrm{~mL} 10 \mathrm{mM}$ Tris buffer solution ( $\mathrm{pH}$ 8.5 ) and then $0.5 \mathrm{~g}$ nano-BN was added. The dark slurrylike product was washed with acetone, and the solvent was removed by centrifuge. The wash-centrifuge step was repeated three times, and finally BN-D-GF/acetone slurry was obtained.

To prepare the BN-D-GF/epoxy FRP composites, a certain amount of E51 was poured into the slurry followed by vigorous stirring for $2 \mathrm{~h}$. The acetone was then removed by rotary evaporation followed by drying in a vacuum oven at $50^{\circ} \mathrm{C}$ for $48 \mathrm{~h}$. The curing agent was then added at the epoxy/curing agent ratio of 100/13 (w/w). The mixtures were mechanically stirred, degassed, and cured at room temperature for $3 \mathrm{~h}$ and postcured at $70^{\circ} \mathrm{C}$ for $2 \mathrm{~h}$. For comparison, a BN/GF/epoxy composite was prepared using the same procedure except that the BN was physically adsorbed onto untreated GF without polydopamine coating.

\subsection{Characterization}

2.3.1. Scanning Electron Microscopy (SEM). Fiber samples were prepared by applying a drop of the particle suspension (in acetone) to a glass slide and then dried overnight. The samples were then sputtered with gold to increase their conductance for SEM observation. Measurements were conducted using Philips XL30 ESEM and Hitachi S-4800 instrument at an operation voltage of $20.0 \mathrm{keV}$ and $0.7 \mathrm{keV}$.

2.3.2. Differential Scanning Calorimetry (DSC). Glasstransition temperatures $\left(T_{g}\right)$ of the neat epoxy resin and FRP composites were measured by using a differential scanning calorimetry (DSC, 200F3, Netzsch, Germany). Samples were heated from 20 to $250^{\circ} \mathrm{C}$ at a heating rate of $10^{\circ} \mathrm{C} / \mathrm{min}$ under nitrogen atmosphere.

2.3.3. Dynamic Mechanical Analysis (DMA). A dynamic mechanical analysis (DMA) was performed with a DMA 2980 Dynamical Mechanical Analyzer in the fixed frequency mode at $1 \mathrm{~Hz}$ with an amplitude of $0.40 \mathrm{~mm}$. The transverse mechanical properties of the composites were measured 
using the DMA. Rectangular DMA specimens measuring 40 $\times 10 \times 3 \mathrm{~mm}$ were sawn and polished to uniform dimensions. The DMA uses a flexural bending deformation to apply stress to the sample. The sample undergoes a sinusoidal oscillation at a fixed frequency.

\subsection{Test of Neat Epoxy Resin and FRP Composites}

2.4.1. Volume Resistivity. A digital high resistance meter (6517A, Keithley, USA) was used to investigate the dc conductivity. Samples with thickness of around $1 \mathrm{~mm}$ were used for measurement. The typical conductivity measurement involves a simple measurement of current under the same ambient atmosphere and a constant voltage $(1 \mathrm{kV})$. The electric current was measured after applying the dc voltage to the specimens for $1 \mathrm{~min}$ according to ASTM D257.

2.4.2. Dielectric Loss. Dielectric properties measurements were performed using an impedance analyzer (Aglient 4294A, USA) with 16451B Dielectric Test Fixture in the frequency range of $10^{2}-10^{7} \mathrm{~Hz}$. All measurements were carried out at room temperature, with voltage amplitude of $0.5 \mathrm{~V}$. The data of impedance and phase angle measured were converted into the relative dielectric constant and dielectric loss, considering the appropriate geometric coefficient.

2.4.3. Dielectric Breakdown Strength. Dielectric breakdown strength was measured using an AHDZ-10/100 alternatingcurrent dielectric strength tester (Shanghai Lanpotronics Corporation, China) according to ASTM D 149-2004. The specimens were placed between two 10-mm-diameter copper ball electrodes and the electrode system containing the measured sample was immersed in the pure silicone oil to prevent the surface flashover. The test voltage was applied across two ball-typed electrodes and was increased until the sample was punctured. Five breakdown tests were repeatedly performed on each specimen. All of the measurements were performed under the same humidity and temperature.

2.4.4. Thermal Conductivity. LFA 447 Nanoflash (Netzsch, Germany) was employed to determine the thermal conductivity according to ASTM E1461. Samples were prepared in cylindrical shape of $130 \mathrm{~mm}$ in diameter and $10-20 \mathrm{~mm}$ in thickness.

2.4.5. Shore D Hardness. Shore D hardness testing was performed according to ASTM D2240 using a Woltest SD 300 equipment.

\section{Results and Discussion}

3.1. Characterization. Figure 1(a) showed that nano-BN was easily aggregated and had relatively round shapes with a fine size of $15-50 \mathrm{~nm}$. Figure 1(b) indicated that when glass fibers were not treated with nano-BN, their surfaces were rather smooth. Dopamine treatment of glass fiber surfaces resulted in rather improved adherence between nano-BN and GF. Different processes of $\mathrm{BN}$ modification of GF were indicated in Figures 1(c) and 1(d), which showed the formation of reinforcement through physical adsorption as well as dopamine adhesion. In Figure 1(c), the smooth surface indicated that less $\mathrm{BN}$ was adhered onto fibers because of the weak physical adsorption. Thus, in BN/GF/epoxy composite, it indicated insufficient adherence to the epoxy matrix, while Figure 1(e) showed that "fiber pullouts" occurred due to the weak interface between epoxy and glass fibers. Additionally, part of nano-BN aggregated and formed microsized particles. However, it was different for specimens that dopamine domains acted as coupling agent and adhered to the glass fiber surfaces (BN-D-GF). In Figures 1(d) and 1(f), BN-DGF showed continuous and rough surfaces due to dopamine treatment as well as nano-BN adhesion. Therefore, this also increased the interaction at the fiber/epoxy matrix interface, while "fiber pullouts" nearly disappeared in BN-D-GF/epoxy composites.

FTIR spectra of the dopamine, GF, and D-GF were shown in Figure 2. The characteristic peak at $1608 \mathrm{~cm}^{-1}$ and $1508 \mathrm{~cm}^{-1}$ found in D-GF further confirmed that dopamine had been polymerized into polydopamine on surface of GF.

DSC was used to investigate the thermal properties of the neat epoxy and FRP composites. The DSC curves shown in Figure 3 indicate an increase in the glass transition temperature of the epoxy resin with the GF loading. The introduction of GF was thought to restrict the mobility of the epoxy molecules. The obvious effect on increasing the glass transition temperature indicated a strong interaction at the molecular level between the epoxy molecules and dopamine or BN layers, which could come only from the dopaminetreated samples (D-GF/epoxy and BN-D-GF/epoxy).

3.2. Dynamic Mechanical Behavior of Composites. Dynamic mechanical properties of FRP composites depend on various factors such as fiber loading, fiber orientation, and the nature of fiber-matrix interface region. Dynamic tests are especially sensitive to all kinds of transitions and relaxation processes of matrix resin and also to the microstructure of the composites.

Dynamic storage modulus $\left(E^{\prime}\right)$ is the most important property to assess the load bearing capacity of a composite material. The variation of storage modulus as a function of temperature of epoxy resin or FRP composites was given in Figure 4 . There was a prominent increase in the modulus of the matrix with the incorporation of glass fibers over the entire region. This may be due to the increase in the stiffness of the matrix with the reinforcement effect imparted by the fibers that allowed for a greater degree of stress transfer at the interface [27]. The maximum $E^{\prime}$ value was exhibited by $\mathrm{D}$-GF/epoxy composite. The value was $376.24 \mathrm{MPa}$, whereas the $E^{\prime}$ value for unreinforced cured neat resin was $214.46 \mathrm{MPa}$ at room temperature. It was clear that the room temperature storage modulus of the matrix enhanced more than $75 \%$ upon the addition of dopamine coating fiber. Previous research had proved that it was due to physical cross-linking caused by the hydrogen bonds between the epoxy molecules and dopamine coating filler, where the catechol groups act as proton donors, while the $\mathrm{C}-\mathrm{O}-\mathrm{C}$ groups in the epoxy act as proton acceptors [28]. For BN-D-GF/epoxy composites, 


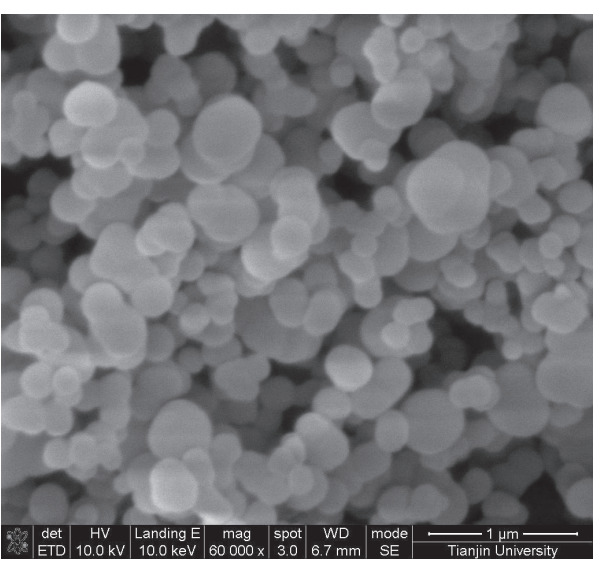

(a)

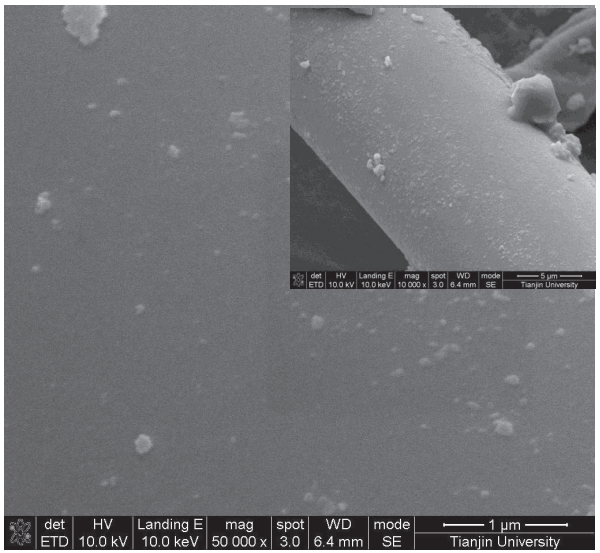

(c)

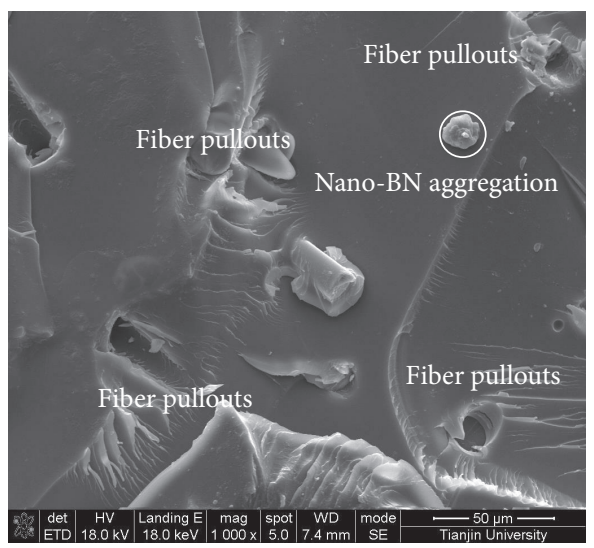

(e)

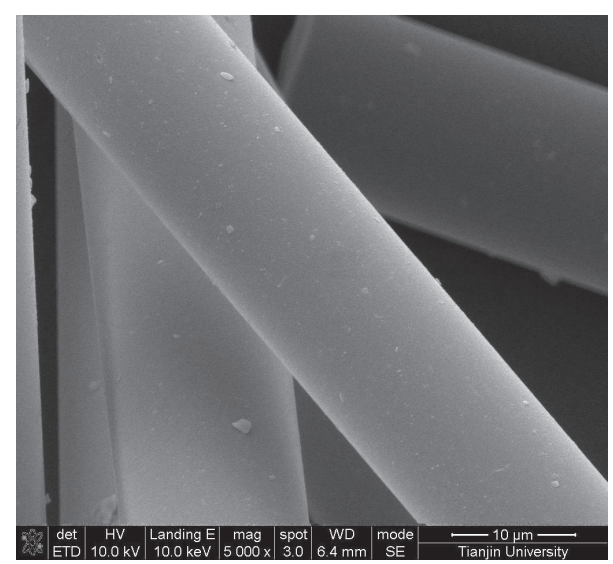

(b)

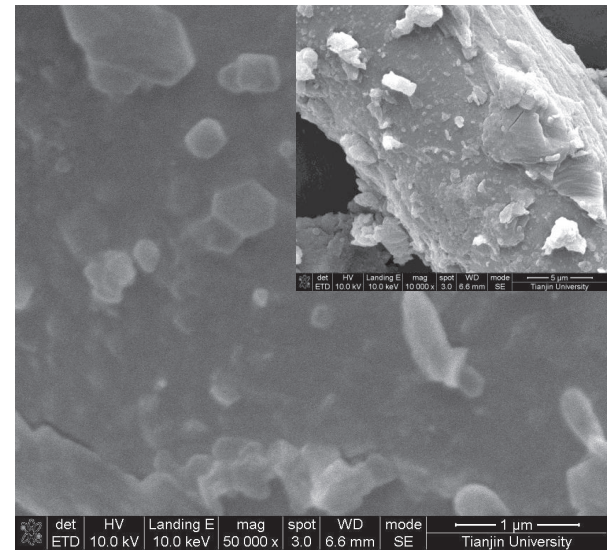

(d)

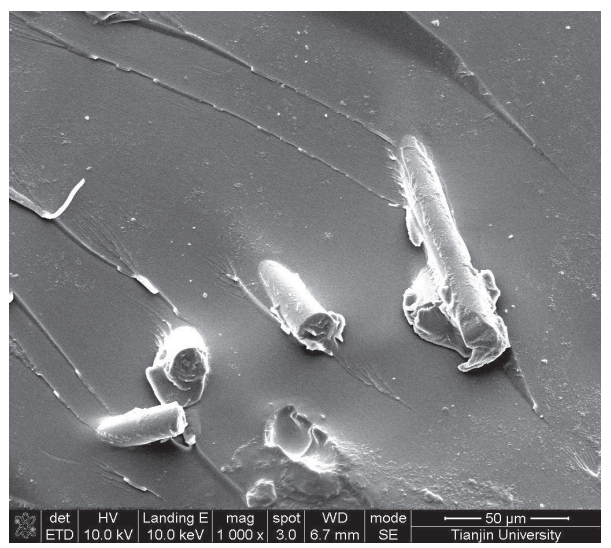

(f)

FIGURE 1: SEM image of BN nanoparticles (a), untreated GF (b), BN/GF (c), BN-D-GF (d), BN/GF/epoxy composite (e), and BN-D-GF/epoxy composite (f).

the value was $337.84 \mathrm{MPa}$, which was $57 \%$ higher than neat resin. As the temperature increases, $E^{\prime}$ decreases and then there was a sharp decline in the $E^{\prime}$ value at the glass transition region. This behavior could be attributed to the increase in the molecular mobility of the polymer chains above $T_{g}$.

Loss modulus $\left(E^{\prime \prime}\right)$ is a measure of the energy dissipated as heat per cycle under deformation. Or, it is the viscous response of the material. Figure 5 showed the trends of variation of loss modulus for the neat epoxy and FRP composites with variation of temperature. From the figure, it was clear that the incorporation of fiber causes broadening of the loss modulus peak. The peak broadening could be attributed to the inhibition of relaxation process within the composites [29]. This might be due to the increase in the number of chain segments as well as more free volume upon fiber addition. The loss modulus value in the transition 
TABLE 1: Tests of neat epoxy and FRP composites.

\begin{tabular}{|c|c|c|c|c|c|}
\hline & $\begin{array}{l}\text { Thermal conductivity } \\
(\mathrm{W} / \mathrm{m} \cdot \mathrm{K})\end{array}$ & $\begin{array}{l}\text { Volume resistivity } \\
\quad\left(\times 10^{16} \Omega \cdot \mathrm{cm}\right)\end{array}$ & $\begin{array}{c}\text { Dielectric loss } \\
\text { tangent }\end{array}$ & $\begin{array}{l}\text { Dielectric breakdown strength } \\
(\mathrm{kv} / \mathrm{mm})\end{array}$ & $\begin{array}{l}\text { Shore D } \\
\text { hardness }\end{array}$ \\
\hline Neat epoxy & 0.252 & 3.00 & 2.53 & 31.1 & 71 \\
\hline GF/epoxy & 0.287 & 2.14 & 2.4 & 24.8 & 78 \\
\hline D-GF/epoxy & 0.308 & 2.67 & 2.14 & 27.3 & 80 \\
\hline BN/GF/epoxy & 0.294 & 3.77 & 2.19 & 25.8 & 79 \\
\hline BN-D-GF/epoxy & 0.393 & 6.86 & 2.01 & 27.7 & 82 \\
\hline
\end{tabular}

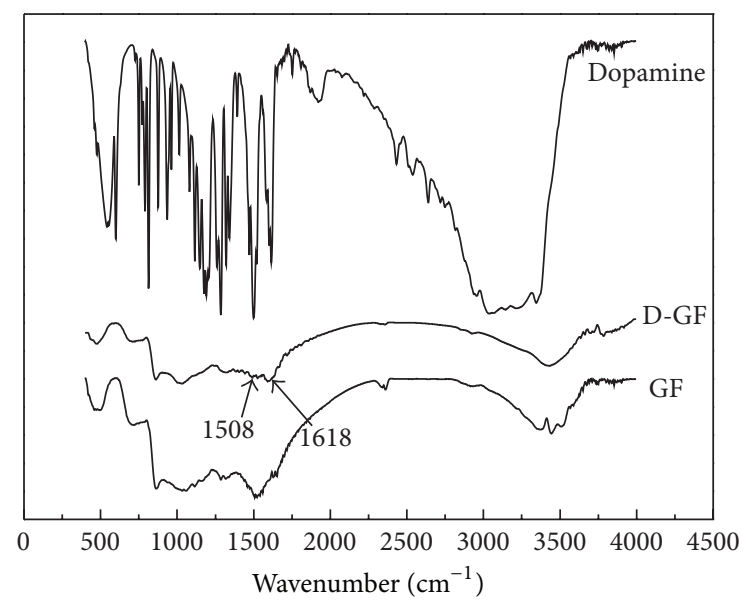

FIGURE 2: FTIR spectra of dopamine and glass fibers.

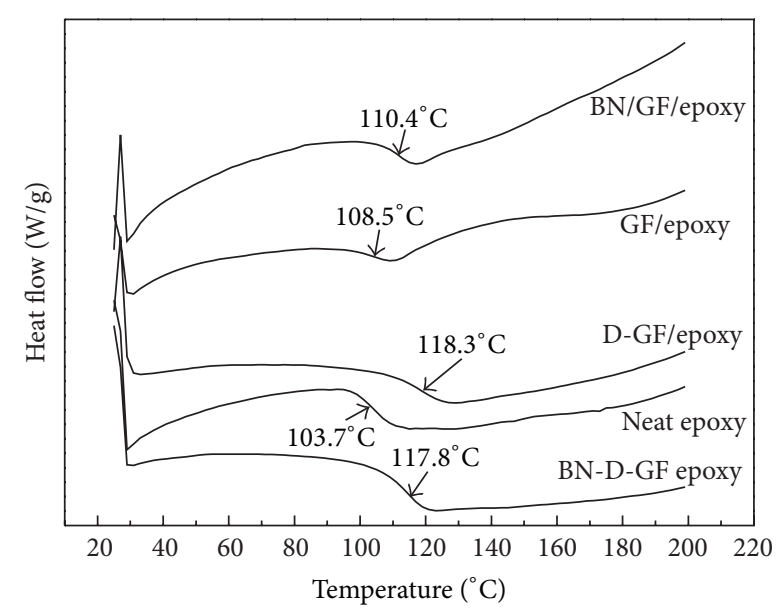

FIGURE 3: DSC curves of neat epoxy resin and FRP composites.

region was much high for composites when compared to the neat resin. The higher modulus at this temperature was due to the increase in internal friction that enhances the dissipation of energy. Additionally, the presence of high modulus glass fibers reduced the flexibility of the material by introducing constraints on the segmental mobility of the polymeric molecules at the relaxation temperatures [30].

3.3. Thermal Conductivity. The measurements of the thermal conductivity of the GF-reinforced epoxy resin were shown

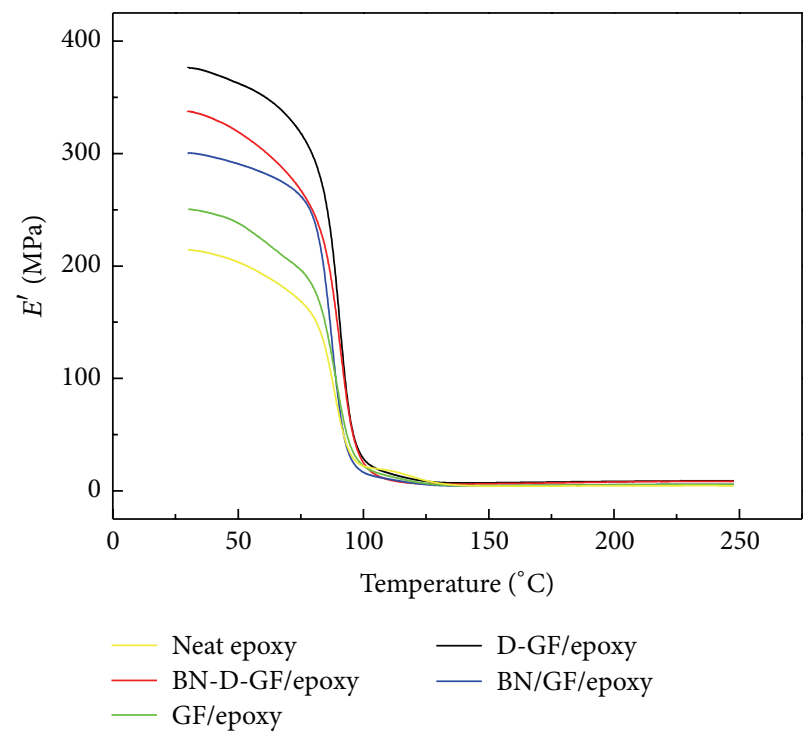

FIgURE 4: Dynamic storage modulus of neat epoxy and FRP composites.

in Table 1. In the same figure, the measurements of the thermal conductivity of the epoxy resin were shown for comparison purposes. It can be seen that reinforcing the polymer with the untreated GF resulted approximately in a $13 \%$ increase in the thermal conductivity. The slight increase may be a result of the relatively better conducting nature of the glass fibers compared to the epoxy-resin matrix. Measurements on BN/GF/epoxy samples show that the physical blending of the $\mathrm{BN}$ with glass fibers had no real effect on the thermal conductivity. It seemed that the discontinuous nature of the nano-BN in the matrix probably nullifies its thermal conductivity. The thermal conductivity of composite increased slightly from $0.287 \mathrm{~W} / \mathrm{mK}$ for untreated GF filled composite to $0.308 \mathrm{~W} / \mathrm{mK}$ for $\mathrm{D}-\mathrm{GF}$ filled composite. The dopamine layer on the GF surface had rendered the surface more organophilic with greater wettability of the epoxy resin. This led to greater interfacial adhesion between the filler and the polymer matrix leading to more efficient heat transfer across the interface, and hence the increase in the thermal conductivity of the D-GF filled composites. Reinforcing the epoxy resin with both BN-D-GF produced a $56 \%$ of thermal conductivity increase. It was possible that the nano-BN has created a better conductive pathway between the glass fibers and polymer matrix and thus the observed increase 


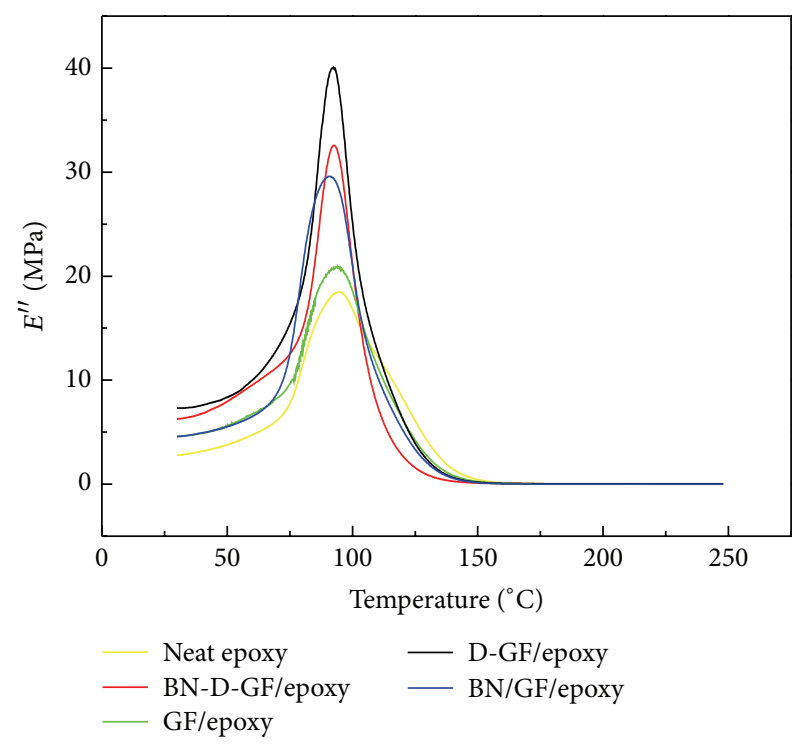

FIgURE 5: Loss modulus of neat epoxy and FRP composites.

in thermal conductivity. A previous study had investigated nanotube-reinforced GF composites and reported that the multiwall carbon nanotubes (C-MWNTs) were repelled by the glass fibers [31]. In a word, thermal conductivity of a FRP composite strongly depends on the efficiency of heat transfer through the interface between the filler and the matrix. Hence, any treatment which enhances the interfacial adhesion between the filler and the matrix could be expected to increase the overall thermal conductivity of the composite as well.

3.4. Electrical Properties. Dielectric breakdown strength of the neat epoxy as well as FRP composites was summarized in Table 1. One can see that (i) all the FRP composites showed lower dielectric strength in comparison with the pure epoxy and (ii) the composites with dopamine-treated GF showed higher values of breakdown strength when compared with the composites with untreated GF. The embedding of inorganic fillers into insulating polymers could introduce different kinds of defects and then result in lower dielectric breakdown strength. There existed two possible reasons causing lower dielectric strength in the FRP composites with untreated GF. The first was incompatible interface between the untreated fibers and epoxy, which can introduce voids in the composites, causing the decrease of dielectric strength. The second was that the agglomeration of the BN could result in electromagnetic interactions among the nanoparticles, which can distort severely the field and in turn cause lower breakdown strength.

The variation of volume resistivity for the neat epoxy as well as FRP composites was summarized in Table 1. It can be seen that (i) the volume resistivity value of the FRP composites with untreated GF was slightly lower than that of the neat epoxy; and (ii) the volume resistivity value of the FRP composites with dopamine-treated GF was higher than that of the neat epoxy. The decreased volume resistivity might originate from the contribution of impurity ions as well as hydroxyl groups, which existed in the surface of glass fibers. Previous results had proved that the surface treatment by means of silane can replace the hydroxyl groups to remove the molecularly absorbed water hydrogen bonded to the surface of the AlN nanoparticles, which reduced the conductivity of the AlN nanoparticles and the nanocomposites [32]. Because the BN ceramic nanoparticles used for FRP composite preparation were dielectric materials like AlN, BN-D-GF/epoxy sample showed that highest volume resistivity value.

The dielectric loss tangent of the neat epoxy as well as FRP composites was summarized in Table 1. One can see that: (i) all the FRP composite samples showed lower values of dielectric loss in comparison with the neat epoxy and (ii) the values of dielectric loss tangent of epoxy nanocomposites with dopamine-treated glass fibers were obviously lower than those epoxy nanocomposites with untreated GF, especially connected with BN. It was proposed that the incorporation of BN-D-GF can result in polymer chain entanglement because of the good dispersion of the nanofiller, which in turn causes immobility of charge carriers or reduction in electrical conductivity, thus causing a reduction of dielectric loss.

3.5. Shore D Hardness. Shore D hardness of the neat epoxy as well as FRP composites was summarized in Table 1. All FRP composites benefited from the GF presence, so that the hardness value was increasing more than $10 \%$. The dopamine coating affected a certain extent, while the value of GF/epoxy and BN/GF/epoxy was slightly increased.

\section{Conclusions}

The present study reported a biomimetic approach to successfully coat a monolayer of PDOPA onto GF. Compared with untreated GF, the PDOPA coating greatly benefited the interfacial interaction, hence leading to enhanced loading amount and homogenous distribution of BN nanoparticles (about $40 \mathrm{~nm}$ ) on GF. BN-D-GF, micronano multiscale hybrid filler, was also demonstrated as an appropriate reinforcement for epoxy resin. The FRP nanocomposites, BN-D-GF/epoxy resin, showed drastically enhanced volume resistance and thermal conductivity. It is expected that the present approach can be extended to preparing a variety of FRP composite systems which include fiber reinforcement, polymer matrices, and functional nanomaterials. This would pave a new way for diverse applications such as adhesive, building materials and opto- and microelectronics devices.

\section{Acknowledgments}

The authors acknowledge the financial support from the National Natural Science Foundation of China (21306139, U1162114), Natural Science Foundation of Tianjin (no. 12JCQNJC06000), Tianjin City High School Science \& Technology Fund Planning Project (20110505, 20130509), Lab Opening Foundation of Tianjin University of Science \& Technology (1103A207), and National Undergraduate Training Programs for Innovation and Entrepreneurship (201310057118). 


\section{References}

[1] L. T. Drazal, Advances in Polymer Science, vol. 75, Springer, Berlin, Germany, 1986.

[2] H. D. Middleton, Composite Materials in Aircraft Structure, Longman, New York, NY, USA, 1990.

[3] A. A. Baker, R. Jones, and R. J. Callinan, "Damage tolerance of graphite/epoxy composites," Composite Structures, vol. 4, no. 1, pp. 15-44, 1985.

[4] W. K. Goertzen and M. R. Kessler, "Dynamic mechanical analysis of carbon/epoxy composites for structural pipeline repair," Composites B, vol. 38, no. 1, pp. 1-9, 2007.

[5] S. Sirivedin, D. N. Fenner, R. B. Nath, and C. Galiotis, "Effects of inter-fibre spacing and matrix cracks on stress amplification factors in carbon-fibre/epoxy matrix composites, Part II: hexagonal array of fibres," Composites A, vol. 37, no. 11, pp. 1936-1943, 2006.

[6] W. K. Goertzen and M. R. Kessler, "Creep behavior of carbon fiber/epoxy matrix composites," Materials Science and Engineering $A$, vol. 421, no. 1-2, pp. 217-225, 2006.

[7] N. Hameed, P. A. Sreekumar, B. Francis, W. Yang, and S. Thomas, "Morphology, dynamic mechanical and thermal studies on poly(styrene-co-acrylonitrile) modified epoxy resin/glass fibre composites," Composites A, vol. 38, no. 12, pp. 2422-2432, 2007.

[8] Y. T. Liao and K. C. Lee, "Effect of interface properties on the static and dynamic properties of unidirectional composites," Journal of Applied Polymer Science, vol. 44, pp. 933-936, 1992.

[9] K. Sever, M. Sarikanat, Y. Seki, and I. H. Tavman, "Concentration effect of $\gamma$-glycidoxypropyl-trimethoxysilane on the mechanical properties of glass fiber-epoxy composites," Polymer Composites, vol. 30, no. 9, pp. 1251-1257, 2009.

[10] M. R. Dadfar and F. Ghadami, "Effect of rubber modification on fracture toughness properties of glass reinforced hot cured epoxy composites," Materials and Design, vol. 47, pp. 16-20, 2013.

[11] F. Tarlochan, S. Ramesh, and S. Harpreet, "Advanced composite sandwich structure design for energy absorption applications: blast protection and crashworthiness," Composites B, vol. 43, pp. 2198-2208, 2012.

[12] M. M. Rahman, S. Zainuddin, M. V. Hosur et al., "Effect of NH2-MWCNTs on crosslink density of epoxy matrix and ILSS properties of e-glass/epoxy composites," Composite Structures, vol. 95, pp. 213-221, 2013.

[13] F. Yavari, M. A. Rafiee, J. Rafiee, Z.-Z. Yu, and N. Koratkar, "Dramatic increase in fatigue life in hierarchical graphene composites," ACS Applied Materials and Interfaces, vol. 2, no. 10, pp. 2738-2743, 2010.

[14] N. Shahid, R. G. Villate, and A. R. Barron, "Chemically functionalized alumina nanoparticle effect on carbon fiber/epoxy composites," Composites Science and Technology, vol. 65, no. 14, pp. 2250-2258, 2005.

[15] G.-W. Lee, M. Park, J. Kim, J. I. Lee, and H. G. Yoon, "Enhanced thermal conductivity of polymer composites filled with hybrid filler," Composites A, vol. 37, no. 5, pp. 727-734, 2006.

[16] M. Ya-nan, L. Guozheng, G. Aijuan, Z. Feipeng, and Y. Li, "Thermally conductive aluminum nitride-multiwalled carbon nanotube/cyanate ester composites with high flame retardancy and low dielectric loss," Industrial and Engineering Chemistry Research, vol. 52, pp. 3342-3353, 2013.

[17] H. B. Cho, M. Mitsuhashi, T. Nakayama et al., "Thermal anisotropy of epoxy resin-based nano-hybrid films containing
BN nanosheets under a rotating superconducting magnetic field," Materials Chemistry and Physics, vol. 139, pp. 355-359, 2013.

[18] S. Sinha Ray and M. Okamoto, "Polymer/layered silicate nanocomposites: a review from preparation to processing," Progress in Polymer Science, vol. 28, no. 11, pp. 1539-1641, 2003.

[19] I. Zaman, Q.-H. Le, H.-C. Kuan et al., "Interface-tuned epoxy/clay nanocomposites," Polymer, vol. 52, no. 2, pp. 497504, 2011.

[20] T. Wan, S. Liao, K. Wang, P. Yan, and M. Clifford, "Multi-scale hybrid polyamide 6 composites reinforced with nano-scale clay and micro-scale short glass fibre," Composites A, vol. 50, pp. 3138, 2013.

[21] M. J. Clifford and T. Wan, "Fibre reinforced nanocomposites: mechanical properties of PA6/clay and glass fibre/PA6/clay nanocomposites," Polymer, vol. 51, no. 2, pp. 535-539, 2010.

[22] N. N. Herrera, J.-M. Letoffe, J.-L. Putaux, L. David, and E. Bourgeat-Lami, "Aqueous dispersions of silane-functionalized laponite clay platelets: a first step toward the elaboration of water-based polymer/clay nanocomposites," Langmuir, vol. 20, no. 5, pp. 1564-1571, 2004.

[23] K. A. Carrado, L. Xu, R. Csencsits, and J. V. Muntean, "Use of organo- and alkoxysilanes in the synthesis of grafted and pristine clays," Chemistry of Materials, vol. 13, no. 10, pp. 37663773, 2001.

[24] N. N. Herrera, J.-M. Letoffe, J.-P. Reymond, and E. BourgeatLami, "Silylation of laponite clay particles with monofunctional and trifunctional vinyl alkoxysilanes," Journal of Materials Chemistry, vol. 15, no. 8, pp. 863-871, 2005.

[25] H. Lee, B. P. Lee, and P. B. Messersmith, "A reversible wet/dry adhesive inspired by mussels and geckos," Nature, vol. 448, no. 7151, pp. 338-341, 2007.

[26] H. Lee, S. M. Dellatore, W. M. Miller, and P. B. Messersmith, "Mussel-inspired surface chemistry for multifunctional coatings," Science, vol. 318, no. 5849, pp. 426-430, 2007.

[27] P. V. Joseph, G. Mathew, K. Joseph, G. Groeninckx, and S. Thomas, "Dynamic mechanical properties of short sisal fibre reinforced polypropylene composites," Composites A, vol. 34, no. 3, pp. 275-290, 2003.

[28] L. Yang, S. L. Phua, J. K. H. Teo et al., "A biomimetic approach to enhancing interfacial interactions: polydopamine-coated clay as reinforcement for epoxy resin," ACS Applied Materials and Interfaces, vol. 3, no. 8, pp. 3026-3032, 2011.

[29] E. M. Woo and J. C. Seferis, "Viscoelastic characterization of high performance epoxy matrix composites," Polymer Composites, vol. 12, pp. 273-280, 1991.

[30] M. A. López-Manchado, J. Biagitti, and J. M. Kenny, "Comparative study of the effects of different fibers on the processing and properties of ternary composites based on PP-EPDM blends," Polymer Composites, vol. 23, no. 5, pp. 779-789, 2002.

[31] M. H. G. Wichmann, J. Sumfleth, F. H. Gojny, M. Quaresimin, B. Fiedler, and K. Schulte, "Glass-fibre-reinforced composites with enhanced mechanical and electrical properties: benefits and limitations of a nanoparticle modified matrix," Engineering Fracture Mechanics, vol. 73, no. 16, pp. 2346-2359, 2006.

[32] S. Kume, I. Yamada, K. Watari, I. Harada, and K. Mitsuishi, "High-thermal-conductivity AlN filler for polymer/ceramics composites," Journal of the American Ceramic Society, vol. 92, supplement 1, pp. S153-S156, 2009. 

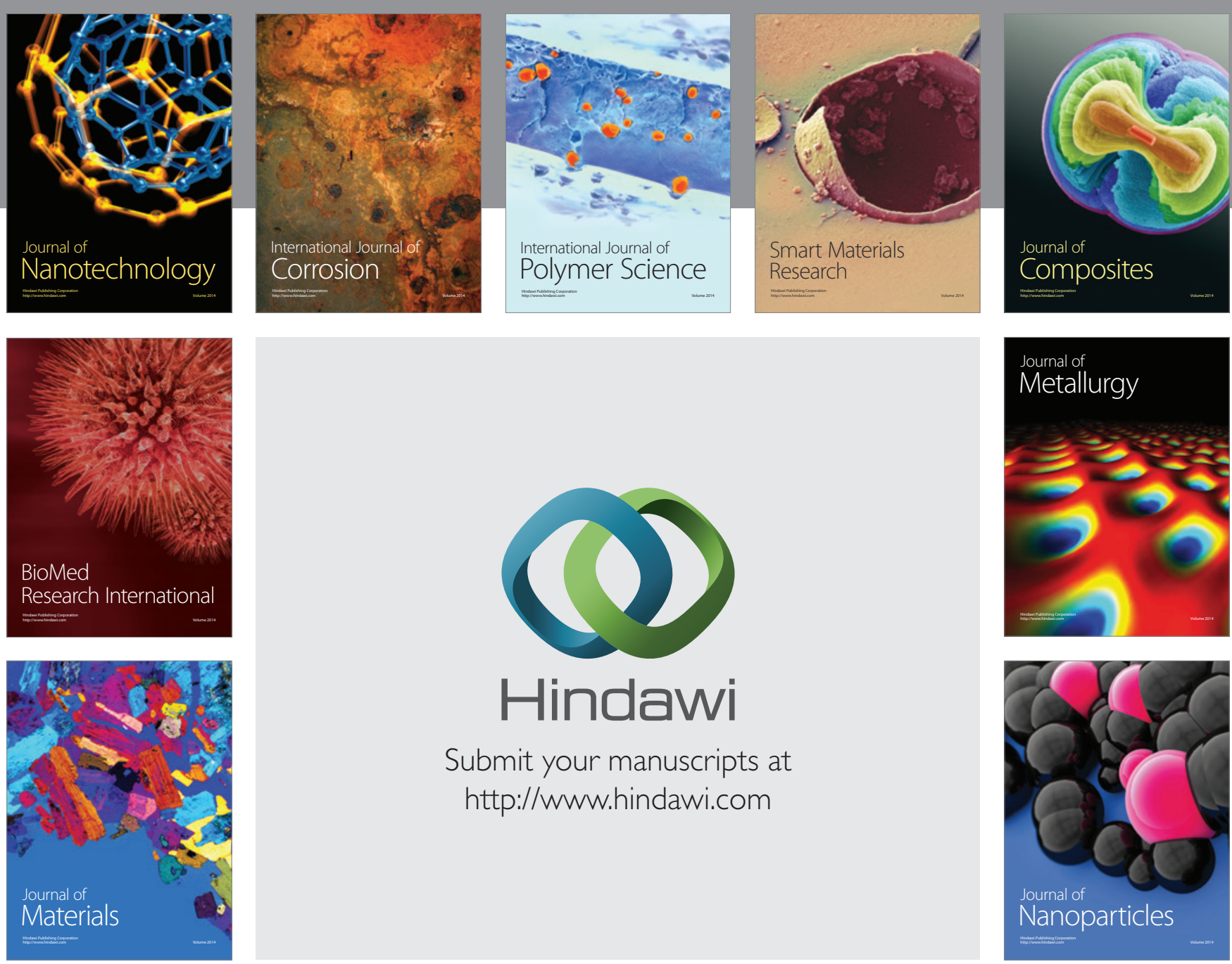

Submit your manuscripts at http://www.hindawi.com
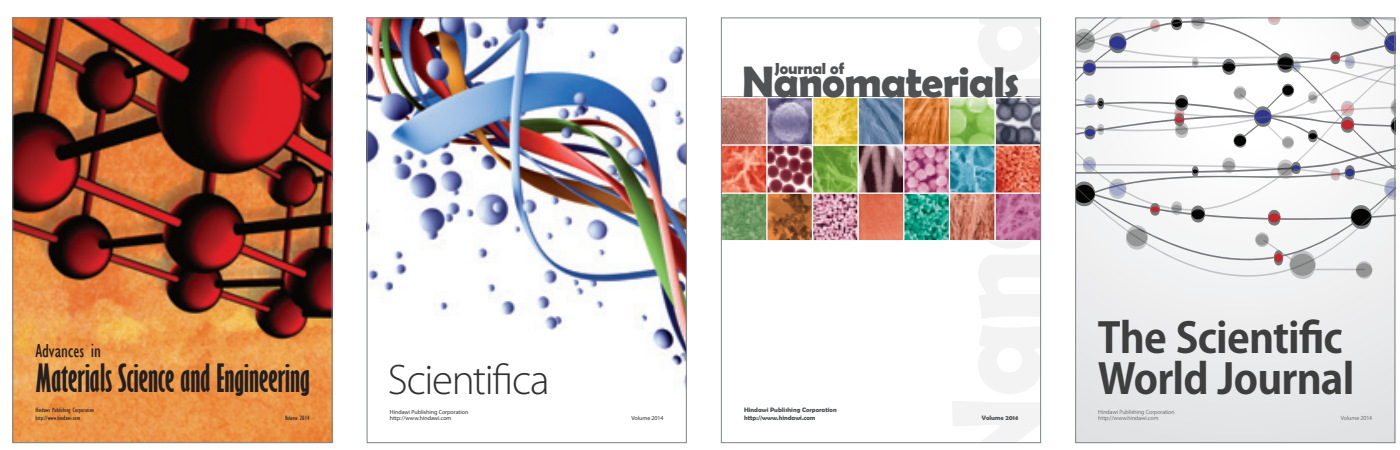

\section{The Scientific World Journal}
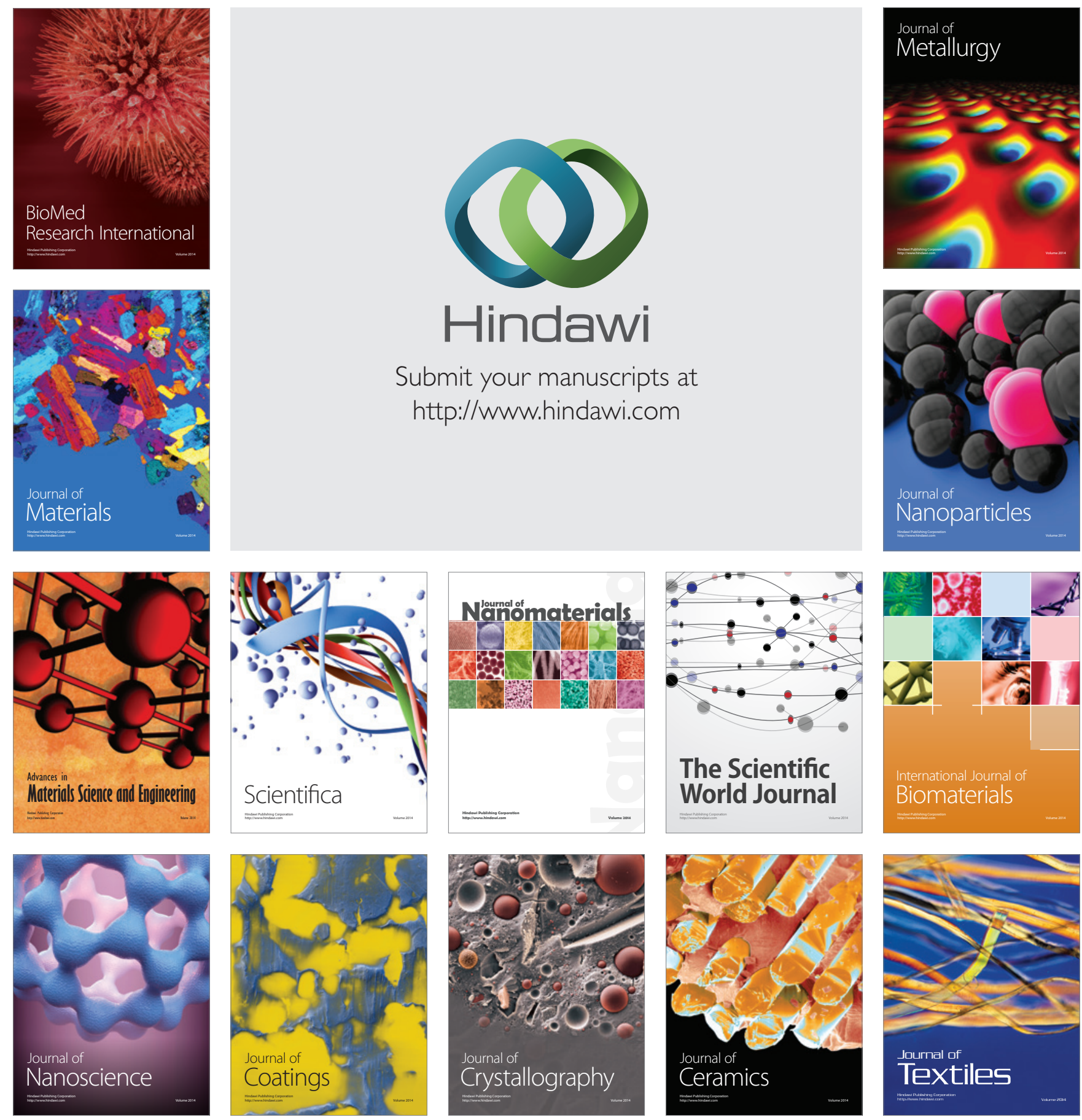